\title{
Theme: Pediatric Pulmonology \& Sleep Medicine
}

ERS statement on protracted bacterial bronchitis in children. (Eur Respir J. 2017;50:pii: 1602139.)

Protracted bacterial bronchitis (PBB) has been increasingly been recognized as a cause of isolated chronic wet cough in under-five children. PBB is commonly misdiagnosed as asthma or wheezing, and leads to inappropriate treatment with bronchodilators and steroids. The awareness regarding the disease entity is poor amongst pediatricians, and there is no epidemiological data from India. The European Respiratory Society consensus statement provides a comprehensive guidelines regarding the diagnosis, evaluation, treatment and follow-up of children with PBB. The article also describes clinical case vignettes and algorithm for evaluation of a child with chronic wet cough.

A meta-analysis of montelukast for recurrent wheeze in preschool children. (Eur J Pediatr. 2017;176:963-9)

Wheezing children below the age of five years are a hetrogenous group of population ranging from virus-induced wheezing to early-onset asthma. The clinical distinctions between these phenotypes can sometimes be difficult. Montelukast is a commonly used drug in under-five children with recurrent wheezing, and is recommended by a few international guidelines. It has been used intermittently as a preemptive treatment to prevent wheezing at the onset of an upper respiratory infection, and also as daily controller therapy. This meta-analysis evaluates the efficacy of montelukast in all phenotypes of pre-school wheezing. The pooled results of studies on intermittent montelukast showed no difference in reducing the number of wheezy episodes, reducing unscheduled medical attendance, and reducing the use of oral corticosteroids. The pooled results of the continuous regimen showed no significant difference in the number of wheezing episodes between the montelukast and placebo groups.

Neuropsychiatric adverse drug reactions in children If initiated on montelukast in real-life practice. (Eur Respir J. 2017;50:pii:1700148.)

The use of montelukast is rampant considering its oral form, and the assumption that it is without any adverse effects compared to the corticosteroids. This retrospective nested cohort study from Canada evaluated the neuropsychatric adverse effects - leading to drug cessation - of montelukast compared to inhaled corticosteroids (ICS) in children with asthma. The incidence of adverse effects was 16\%, most occurring within the first two weeks. Most frequent adverse effects were irritability, aggressiveness and sleep disturbances. The relative risk $(95 \% \mathrm{CI})$ of neuropsychiatric adverse effects associated with montelukast over ICS was 12 (2-90). These data are comparable to the results from VigiBase, WHO Global Individual Case Safety Report (ICSR) database.

Metabolic consequences of obstructive sleep apnea in adolescents with obesity. (Child Obes. 2017;13:102-10)

Sleep disordered breathing in children with obesity is under recognized in children and adolescents. Both obstructive sleep apnea (OSA) and hypoventilation are described, and can occur in combination as well. It can lead to various complications such as sleep fragmentation, pulmonary hypertension, systemic hypertension and metabolic syndrome. Dyslipidemia, insulin resistance, cardiovascular disease risk, nonalcoholic fatty liver disease, and difficulty with weight loss are well described in adults but data in children and adolescents are sparse. This meta-analysis of 10 studies concluded that although obesity leads to increased metabolic risk, OSA appears to independently increase metabolic impairment. Adolescents with obesity should be frequently screened for OSA to determine need for treatment and reduce this metabolic burden.

Position paper for the use of a home sleep apnea test in children. ( $J$ Clin Sleep Med. 2017 Aug 29. pii: jc-1700431)

The gold standard for diagnosis of obstructive sleep apnea/ hypopnea syndrome $(\mathrm{OSA} / \mathrm{H})$ in children is a polysomnography. As this involves performing a sleep study in a sleep laboratory, miniature versions have been manufactured for performing sleep studies at home, especially in resourcepoor settings. This is fraught with problems such as poor signal quality, lack of monitoring and limited channels. The market of home sleep apnea testing devices has increased many-folds with most manufacturers claiming easy testing at home. The American Academy of Sleep Medicine (AASM) has suggested its use in adults for screening in uncomplicated OSA/H. The current position paper by the AASM has recommended against home sleep apnea test for the diagnosis of OSA/H in children. Testing in a proper sleep laboratory would remain as the gold standard but the individual circumstances of the patient, available diagnostic tools, accessible treatment options, and resources would also need to be considered.

Ankit PARAKh ankitparakh102@gmail.com 